\title{
Multiple Payload Adapters; Opening the Doors to Space
}

\author{
John E. Higgins, Brandon J. Arritt, Eugene R. Fosness, \\ Peter M. Wegner, and Steven J. Buckley
}

\author{
U.S. Air Force Research Laboratory \\ Space Vehicles Directorate \\ 3550 Aberdeen Ave. SE \\ Kirtland AFB, NM 87117-5776 \\ John.Higgins@kirtland.af.mil \\ (505) 846-5087 \\ Brandon.Arritt@kirtland.af.mil \\ (505) 846-9389 \\ Eugene.Fosness@kirtland.af.mil \\ (505) 846-7883 \\ Peter.Wegner@kirtland.af.mil
}

(505) 853-3486

\author{
$T R W$ \\ 3550 Aberdeen Ave. SE \\ Kirtland AFB, NM 87117-5776 \\ Steven.Buckley@trw.com \\ (505) 853-7799
}

\section{Abstract}

In order to increase the number of satellites that can be flown with reduced costs, Multiple Payload Adapters (MPAs) are needed to take advantage of excess payload capability on launch systems. This paper will discuss the development of several MPAs at the Air Force Research Laboratory Space Vehicles Directorate in support of current and future Air Force and Department of Defense requirements. The adapters are being designed using state-of-the-art manufacturing processes, launch vibration isolation, and low-shock separation technology that can accommodate multiple satellite configurations. The MPAs can deploy multiple satellites, in a large range of sizes ( $15 \mathrm{~kg}$ to $1000 \mathrm{~kg}$ ), depending on the design configuration. The MPAs are being developed to support the Minuteman and Peacekeeper derived space launch vehicles, the Evolved Expendable Launch Vehicle, and the Space Shuttle. The successful development of these adapters will greatly reduce the cost of launching satellites into orbit by allowing for the efficient use of currently unused payload margins. These MPA concepts maximize the opportunity for low-budget satellites to be manifested for launch, and are being proposed to fly as early as 2003. Additionally, work has begun to standardize adapter configurations and connections across multiple launch vehicles to provide reduced flight integration costs and greater opportunities for inclusion of small experiments on larger missions.

Table of Contents

1. INTRODUCTION

\section{EELV SECONDARY PAYLOAD ADAPTER (ESPA) \\ 3. UNIVERSITY NANOSAT PROGRAM \\ 4. MiNOTAUR MULTIPLE PAYLOAD ADAPTER \\ 5. FUTURE DEVELOPMENTS \\ 6. SUMMARY}

\section{INTRODUCTION}

Despite growing worldwide interest in small satellites, launch costs continue to hinder the full exploitation of small satellite technology. In the United States, the Department of Defense (DOD), National Aeronautics and Space Administration (NASA), other government agencies, commercial companies, and many universities use small satellites to perform space experiments, demonstrate new technology, and test operational prototype hardware. In addition, the DOD continues to study the role of small satellites in fulfilling operational mission requirements. However, United States (US) government agencies are generally restricted to the use of US launch vehicles, thus eliminating many affordable launch opportunities. Additionally, many small satellite users are faced with shrinking budgets that limit the availability of "affordable" launch opportunities. Because of these factors, numerous satellites are shelved as they wait for space lift. Some of these were eventually mothballed as their launch window passed, wasting millions of research and development dollars. In order to increase the number of spacecraft that can be flown with a small, fixed budget, the Air Force Research Laboratory Space Vehicles Directorate is developing low-cost solutions for the small satellite launch problem. 
There are two primary benefits to using this untapped launch capability. First, it becomes possible to dramatically increase the amount of experiments conducted in space. This additional research can then provide many tangible benefits. For example, many small satellites serve as technology demonstrators for future large DOD satellites. By demonstrating this technology on a small satellite, it can effectively reduce the risks and costs inherent to these massive Air Force and military programs. The second benefit of tapping this previously unused lifting capacity is that it can work to dramatically reduce launch costs. With these Multiple Payload Adapters (MPA), we will be able to place multiple payloads on a single launch vehicle rather than spreading them out over several launches. These MPAs will save the Air Force millions of dollars in spacelift costs by using our space launch capability in a more efficient manner, creating an opportunity for several programs to share launch costs. Additional benefits include potential for improved satellite launch environments with pre-qualified mechanical vibration and shock regimes based on features integrated directly into the standard adapter structure, and simplified payload integration through standardized mechanical and electronic connection designed for optimum flexibility and minimum mass.

One major requirement for these opportunities for improved access to space to be realized is that spacecraft design teams begin to develop their satellites to the specifications of these adaptors. Major program offices, such as the Evolved Expendable Launch Vehicle (EELV) System Program Office (SPO) and the Space and Missile System Center (SMC) have expressed a willingness to incorporate these adaptor features into their program design requirements, giving them a significant increase in the probability of a timely launch. Each of the payload adaptors under development incorporates "standard" mechanical and electrical interfaces in order to facilitate this design process. As an example, all the EELV payloads are required to interface with a stipulated Payload Interface. The smaller and less costly secondary payloads should be held to the same standard to ensure them a greater probability of getting to space.

The Air Force Research Laboratory Space Vehicles Directorate AFRL/VS has been working with several commercial companies to develop innovative technologies that can provide solutions to these space lift shortfalls. In order to establish flight heritage for the following systems, the AFRL has arranged several flight demonstrations to facilitate the transition of these technologies to the commercial industry. A discussion of these projects is included.

\section{EELV SECONDARY PAYLOAD ADAPTER (ESPA)}

In 1995, the Air Force's Space Test Program (STP), managed under the Space and Missile Test and Evaluation Directorate of the Space and Missile Center (SMC Det 12/ST) identified large unused payload margins on the majority of DOD's Evolved Expendable Launch Vehicle (EELV) manifests. In some cases this unused lifting capacity approached $3628 \mathrm{~kg}(8000 \mathrm{lb})$. As a result of these findings, the STP advocated using this excess margin for the deployment of secondary payloads. The STP has teamed with the AFRL/VS to develop a low-cost solution that will exploit this opportunity, refer to Peter M Wegner, et al.

The EELV Secondary Payload Adapter (ESPA) is designed to deploy six radially mounted, $181 \mathrm{~kg}$ (400 lb) secondary payloads and a $6800 \mathrm{~kg}(15,000 \mathrm{lb})$ primary. The ESPA is targeted for an EELV flight in FY06. Qualification tests are currently being conducted on the first ESPA adaptor at the AFRL test facilities in Albuquerque, NM. Figures 1 through 3 illustrate the ESPA concept.

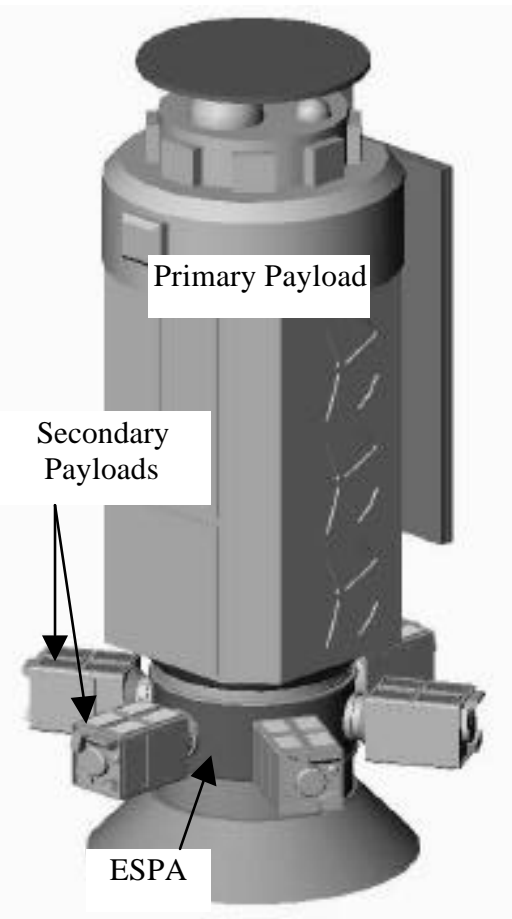

Figure 1. ESPA on the Launch Vehicle Stack.

The ESPA is an adapter ring that will be installed between the EELV final stage and the primary payload. The ESPA cylinder incorporates the 157.7 
$\mathrm{cm}$ (62.01 in) EELV Standard Interface Plane, on both the fore and aft plane, allowing it to mount onto Lockheed Martin's Atlas V and Boeing's Delta IV launch vehicles, as well as payloads that are specifically designed to deploy from the EELV. In addition, the ESPA features six standardized $38 \mathrm{~cm}$ (15 in) diameter secondary payload mounting locations.

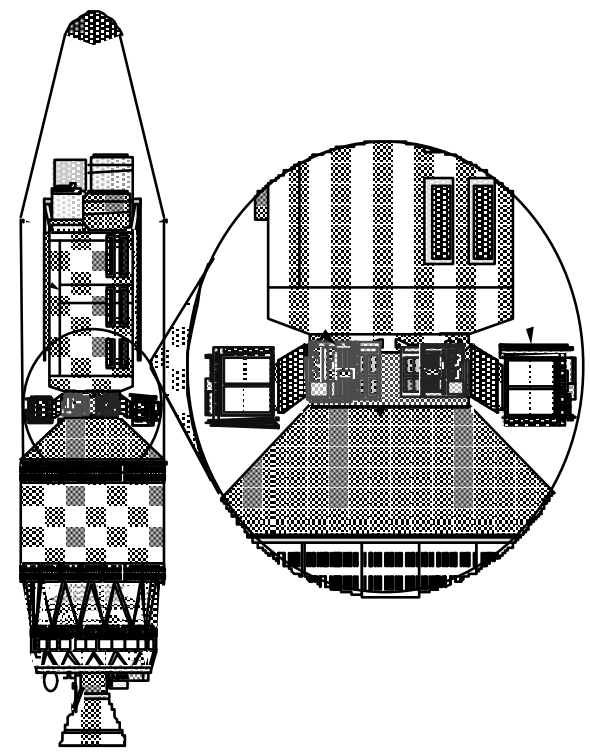

Figure 2. EELV Adapter Concept.

When integrated under the primary payload, and incorporating the optional primary payload isolation system, the ESPA raises the primary payload $76 \mathrm{~cm}$ (30 in). This change in height, when unaccounted for, can result in dramatically increased loading on the primary payload, as well as altered vibrational modes in the entire launch vehicle. In order to accommodate this change in height, and to reduce its effects on the primary and the launch vehicle, the ESPA structure is designed to be stiff in all directions. This approach minimizes the effect the change in height has on the rocking frequency of the primary payload. The high stiffness and the use of the EELV Standard Interface Plane make the ESPA nearly transparent to the primary payload, as well as the rest of the launch vehicle.

The entire ESPA cylinder, including primary and secondary payload mounting flanges, is machined from a single billet of 7075 Aluminum. This results in dramatically reduced labor compared to the alternative of bolting these flanges to the ESPA cylinder. It also greatly reduces the risk, uncertainty, part count, and lead-time associated with the bolted or bonded joints. This design approach has reduced the cost of fabricating the ESPA structure from nearly $\$ 300 \mathrm{~K}$ to about $\$ 75 \mathrm{~K}$ when compared to a similar design using composite materials. Additionally, the use of an integrally machined aluminum structure precludes the necessity for acceptance testing of the structure. This results in an additional cost savings of over $\$ 150 \mathrm{~K}$. Integrally machining the ESPA from a single aluminum billet reduces the recurring manufacturing costs by nearly $\$ 450 \mathrm{~K}$ and saves nearly six months in lead-time, compared to a similar design using composite materials.

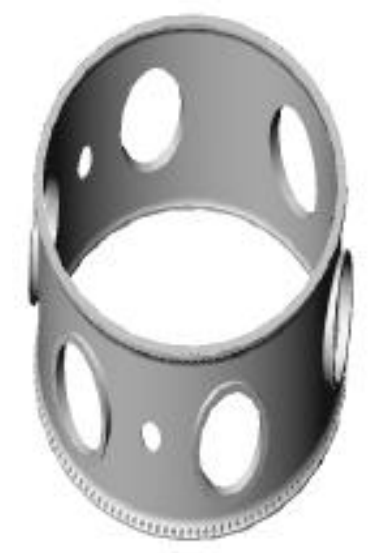

Figure 3. EELV Secondary Payload Adapter (ESPA).

Another innovative technology that ESPA will use for its payloads is whole-spacecraft isolation. During the past decade, millions of dollars have been lost due to satellite malfunctions, resulting in total or partial mission failure. Many of these failures have been linked to launch vibration loads. The AFRL/VS will reduce these launch vibration loads on the satellites via two mechanisms, Soft-Ride for Small Satellites (SRSS) and shock rings.

The SRSS, developed by the AFRL/VS and CSA Engineering, Incorporated of Mountain View, CA is the world's first successfully flown whole-spacecraft isolation system. It has been successfully demonstrated on two different launch vehicles and many payloads. The SRSS has flown on Taurus three times, and on Minotaur twice. The SRSS is a low-risk, passive device that can be tailored for the needs of a particular launch. This tailoring involves both the frequency response of the isolation system, as well as the direction of damping (axial, lateral, or both).

The second flight using the SRSS was launched on a Taurus launch vehicle. Data from that flight is shown in Figure 4. As demonstrated by this graph, the SRSS was able to achieve an overall $50 \%$ RMS 
acceleration reduction, seen from all loading conditions, while reducing the structural vibrations at the peak accelerations by a factor of five.

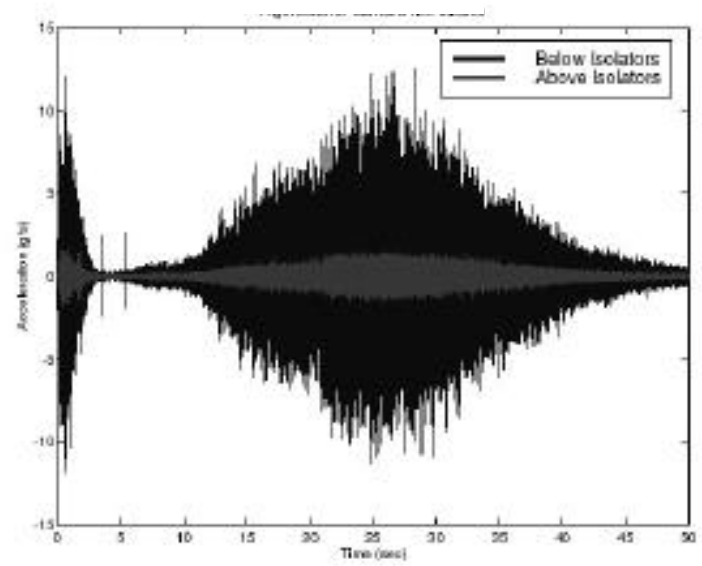

Figure 4: Flight Data from SRSS $2^{\text {nd }}$ Flight..

The SRSS is optimized for frequencies below 100 hertz and can be used for vibration isolation on the primary payload. The other whole-spacecraft isolation system, shock rings, will be used for the secondary payloads. Shock rings are optimized for frequencies above 150 hertz (frequencies between 100 and 150 hertz have historically not been problem areas). The secondary payloads, because of their horizontal mounting, require additional stiffness of the shock rings, as opposed to the flexibility of the SRSS. Much like the SRSS, shock rings can be tailored to more effectively isolate specific frequency ranges.

In addition to whole-spacecraft isolation, the ESPA will incorporate low-shock separation systems to further decrease adverse loading on the payloads. Impulsive loads from pyrotechnic separation systems are another potential cause of irreparable satellite damage and mission failure. By employing nonpyrotechnic separation systems, it is possible to reduce these shock loads on the spacecraft by more than and order of magnitude. The separation systems for the ESPA will be designed to provide an interface between the launch vehicle and payload and will meet structural and safety requirements while having minimal impact on the payload with regard to shock, tip-off, deployed mass, and surface area coverage.

The ESPA is currently undergoing static qualification testing on the AFRL/VS's test stand (as shown in Figure 5). The three objectives of this testing are verify the ESPA stiffness, verify the ESPA strength margins, and validate modeling procedures. The computer-controlled test stand is capable of commanding 20 servo-hydraulic actuators and reading 256 channels of analog data.

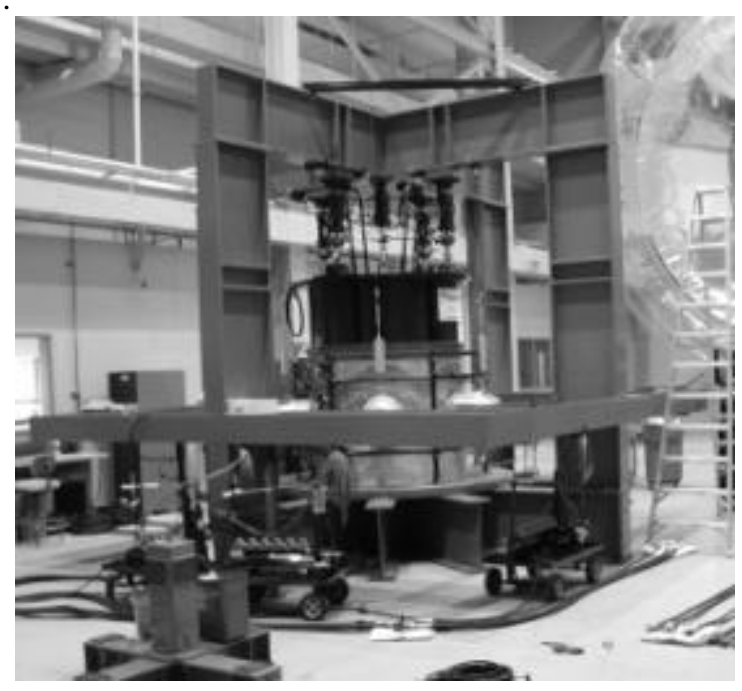

Figure 5. Test Stand at AFRL/VS.

\section{UNIVERSITY NANOSAT PROGRAM}

A promising way to perform many space missions is to use clusters of microsatellites that operate cooperatively to perform the function of a larger, single satellite. The University NanoSat program is a collaborative effort between the AFRL/VS, the Air Force Office of Scientific Research (AFOSR), the Defense Advanced Research Projects Agency (DARPA), the Space Test Program (STP), and NASA/GSFC to explore this shift in paradigm. The program consists of multiple nanosatellites (nanosats) designed and built by US universities that are baselined to deploy from the Space Shuttle via the Shuttle Hitchhiker Experiment Launch System (SHELS), refer to Lt. Carrie Brackett, et al. The current NanoSat flight compliment, dubbed Nanosat2 , consists of three satellites designed and built by Arizona State University, the University of Colorado at Boulder, and New Mexico State University. The Nanosat-2 payload is scheduled to fly on the Space Shuttle in late CY 03. A schematic of this configuration is shown in Figure 6. 


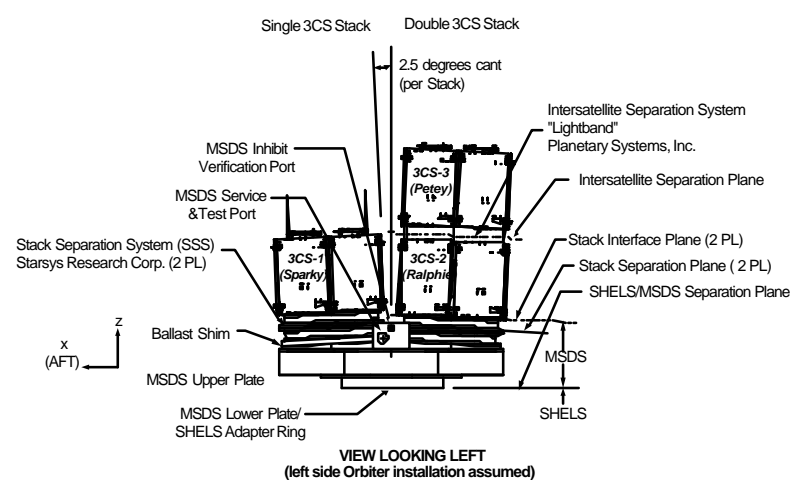

Figure 6: Nanosat-2 General Arrangement.

The AFRL/VS is tasked with developing the integrating structure and separation systems needed for the deployment of the nanosatellites. The integrating structure consists of an aluminum platform with two mounting locations. Each mounting location can support a stack of one or more nanosatellites. The integrating structure also provides the interface to the SHELS, and the whole system is installed in the Shuttle Orbiter Payload Bay. The integrating structure was designed so that each stack of nanosatellites is canted $2.5^{\circ}$ from the Zdirection, giving the stacks a total divergence of $5^{\circ}$, (as shown in Figure 6). This divergence provides a relative separation velocity between the two stacks when they are launched simultaneously from the integrating structure.

To deploy the satellites, the AFRL is developing separation systems with Starsys Research Corporation, and Planetary Systems Corporation. Both systems will employ non-pyrotechnic, lowshock separation techniques. Low-shock separation was required for this program because of the close proximity of the critical satellite components to the separation event, as well as the potential to experience multiple device firings. Some satellites on the Nanosat-2 mission will experience shock from three distinct separation events. Therefore, lowshock separation systems must be utilized in order to prevent damage that these systems could otherwise cause to the satellites. Figure 7 shows the MultiSatellite Deployment System (MSDS) integrating structure and the Stack Separation Systems (Starsys Research Corp). Figure 8 shows the Lightband Separation System (Planetary Systems Corp).

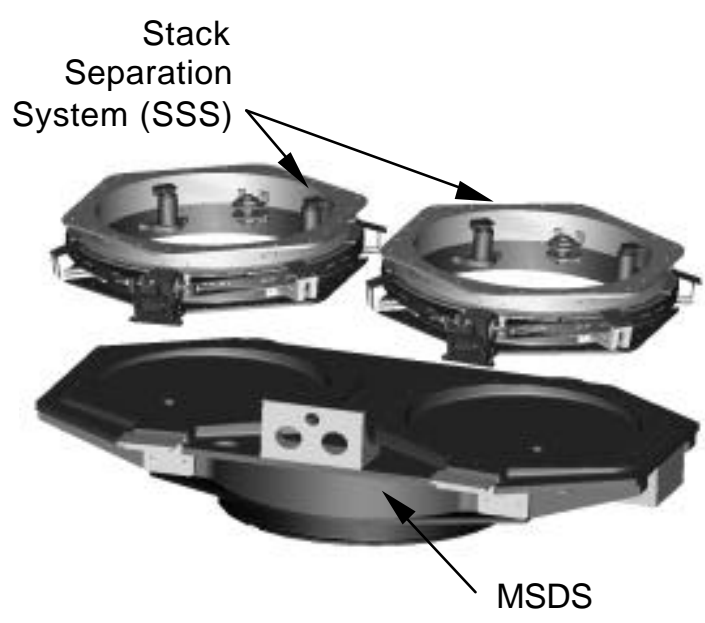

Figure 7: Integrating Structure and Stack Separation System.

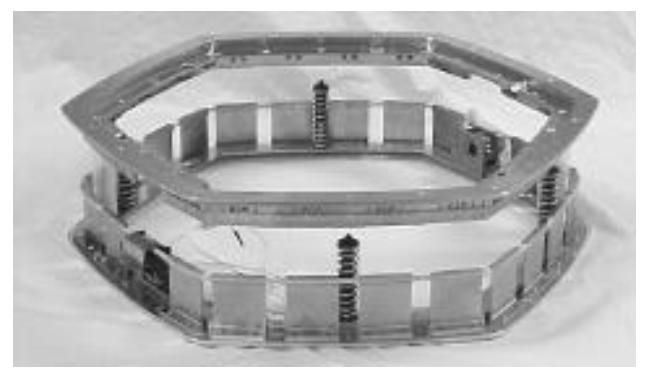

Figure 8: Intersatellite/Lightband Separation System.

The nanosatellites deploy and are placed in their proper orbit through the following three separation events. First, the entire NanoSat system (to include the integrating structure and two stacks of satellites) separates from the SHELS via a separation system developed by NASA/GSFC. Once clear of the Shuttle, each stack of satellites is simultaneously deployed from the integrating structure via the Stack Separation Systems. A significant latency period allows the Shuttle Orbiter ample time to obtain a safe distance, thus avoiding any potential re-contact hazards. After the stacks deploy, the Lightband systems separate the individual satellites, at a time determined by the Universities who developed the satellites.

\section{MinOtaur Multiple PAYlOAd AdAPTER} The "Minotaur Multiple Payload Adapter" program with Applied Aerospace Structures Corporation (AASC), Stockton, CA will design, fabricate, test, and flight qualify a fully re-configurable MPA. The MPA (as shown in Figure 9) will be designed to accommodate 2 to 10 small spacecraft for the 
Minotaur launch vehicle. This is a jointly funded effort between the AFRL/VS and the SMC Det 12/RP, refer to Eugene R. Fosness, et al.

The primary goal of this program is to design a generic, flight-qualified, reconfigurable MPA for various mission scenarios and rapidly changing flight manifests. By using a "building block" approach and post-assembly inserts and routing, AASC will be able to quickly piece together a custom-made MPA for practically any mission scenario. In addition, AASC will be able to rapidly reconfigure the MPA if there occurs a sudden, last minute change in launch manifest.

The Minotaur MPA will incorporate the use of innovative joining techniques, low-shock, nonpyrotechnic separation systems, and whole-spacecraft isolation. The separation systems and isolation are of the same type being used on ESPA.

As illustrated in Figure 9, there are multiple configurations for the MPA. It is anticipated that using these configurations will greatly enhance the capability of the MPA to carry a wide variety of payloads with a negligible influence on the total cost of the program. All configurations will use the same structural members and jointing techniques. The top configuration is the basic "triad" (a fourth vertical could be added to produce a "quad" arrangement). The vertical panels connect to form a torque box in the middle of the structure. To adjust for the needs of a particular payload package, the angle between any two walls can be varied. In this configuration there is more flexibility in maneuvering payloads, thereby optimizing the center of gravity location (CG) and mass characteristics of the MPA, as a whole. The bottom illustration of Figure 9 shows the "cantilevered" baseline. Initial analysis showed the "triad," and its adjunct the "quad," to be optimized for larger numbers of small satellites. However, they fell short in accommodating two larger satellites. In order to retain the ability to launch two satellites, the "cantilevered" option has been carried forward. Again, the inclusion of both designs has not added significantly to the total cost of the program because both families of configurations use the same basic building blocks and design methodology.
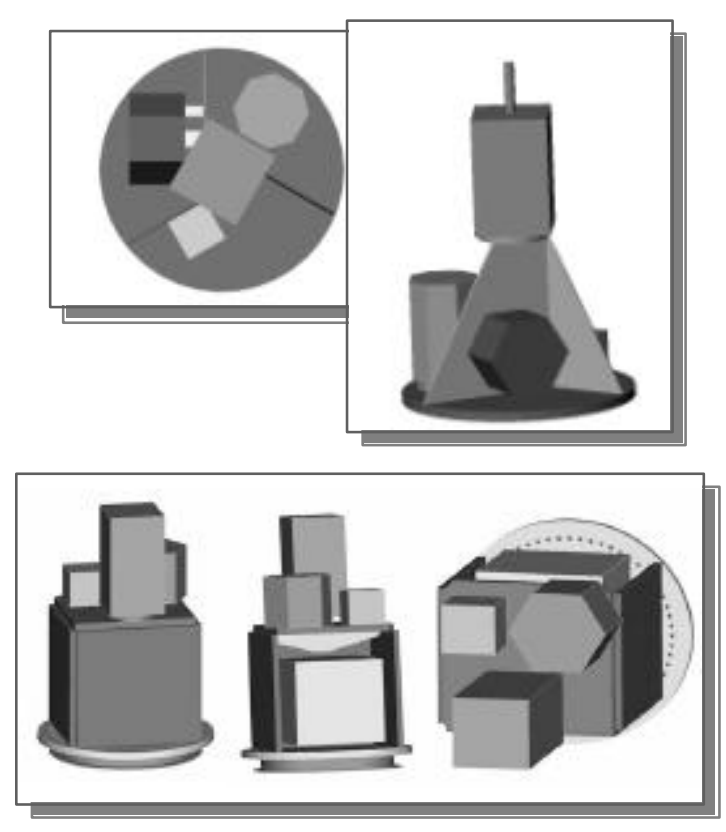

Figure 9. Multiple Payload Adapter Concepts for Minotaur.

This MPA, much like the ESPA, will undergo flight qualification testing in the test facility at the AFRL/VS (Figure 5) during the summer of 2002. Like the ESPA, the three objectives of this testing are to verify stiffness, verify strength margins, and validate modeling procedures.

\section{FUTURE DEVELOPMENTS}

Payload adapters represent a viable way to efficiently use the full spacelift capacity of a launch vehicle. The AFRL has developed a variety of innovative ways to adapt various satellite payloads to specific launch vehicles such as the Minotaur. The next step in the development of these systems is to "polish" the design and add useful features such as payload isolation integrated directly into the structure and advanced systems such as Multi Functional Structures (MFS) cabling for mass reduction. An important improvement to the spacecraft protection program is to include spacecraft isolation as an integral part of the structure of the adapter. Current isolation systems developed by the AFRL/VS are configured as appliances that "insulate" the payload from launch vehicle loads. These systems are highly effective but require additional effort to integrate them with the payload interface and the separation system. If the payload isolation can be integrated into the structure of the adapter, large cost savings would result in manufacturing and the labor required to integrate the adapter to the launch vehicle. The MFS cabling represents a way to reduce the mass of 
the required cables to less than one-tenth the mass of a conventionally configured cable. Additionally, the MFS cabling greatly reduces the labor costs associated with cable harness build-up and installation.

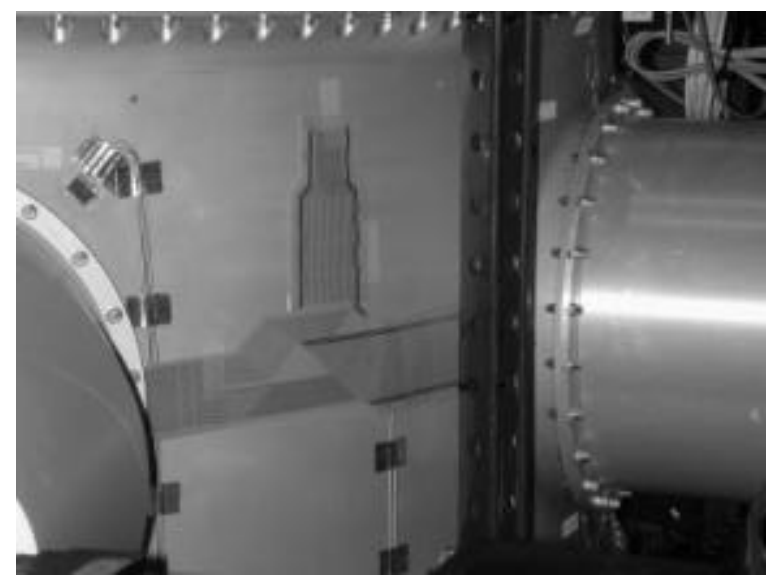

Figure 10. Multiple Functional Structures Cable on Single Curvature ESPA Adapter Surface.

Another initiative is to develop a standardization of the mechanical and electrical interfaces across various launch vehicle configurations. This goal is critical to ensuring maximum use of these systems and represents a necessary step to ensure efficient use of these devices. For example, the ESPA 38-cm (15inch) secondary Payload Interface $(\mathrm{P} / \mathrm{I})$ is an ideal size to support small R\&D satellites. Small satellite programs can leverage the extensive work that was done to develop this ESPA interface (including complimentary low-shock separation systems) by choosing the 15-inch P/I as the standard for their project. This allows this satellite project to save funds by avoiding costly redesign of the P/I and separation system components. Similar efficiencies can be gained by standardization to the $98.55-\mathrm{cm}$ (38.8-inch) Minotaur interface for small launch vehicles and standardization to the $157.5-\mathrm{cm}(62.01-$ inch) EELV interface for larger launch vehicles. A final focus of the adapter program is to develop innovative structures that allow Space Shuttle payloads to fly on an Expendable Launch Vehicle (ELV). Shuttle payloads are designed with load paths that smoothly integrate with the typical Shuttle trunnion or cradle. These payloads typically cannot launch on an ELV without major redesign to account for the fact that the ELV load path is through the base of the payload. An adapter that can transfer the launch vehicle loads from the ELV payload interface to the Shuttle-type trunnion hard points will allow expedient launches of Shuttle payloads using an existing ELV. The size, strength, and mass requirements of this adapter suggest that an innovative composite design might offer the best solution.

\section{SUMMARY}

The MPA technologies that the AFRL and their team members are developing will have a major impact on future military and commercial spacecraft programs by providing a fast and inexpensive way of launching multiple small payloads. The MPA technology allows the use of excess launch capacity that is currently not being used. For example, an EELV lifting a single large DOD satellite may have several thousand kilograms of excess capacity. The ESPA can take advantage of this excess capacity, saving $\$ 15 \mathrm{M}$ to $\$ 45 \mathrm{M}$ in launch costs. The MPAs are being designed to have minimal impact to the primary payload and provide improved flight environments through the use innovative state-of-the-practice component systems such as vibration isolation and low-shock non-pyrotechnic separation.

\section{REFERENCES:}

Brackett, Carrie Lt., et al. "AFRL's Multi-Satellite Deployment System (MSDS).” SSC01-XI-5

Fosness, Eugene R., et al. "Multiple Payload Adaptors for Low Cost Access to Space." AIAA2001-4584

Wegner, Peter M., et al. "EELV Secondary Payload Adapter (ESPA): Providing Increased Access to Space."

\section{BIOGRAPHIES:}

1Lt. Brandon Arritt is a research engineer with the Air Force Research Laboratory's Space Vehicles Directorate. His work focuses on developing next generation launch vehicle structures that will greatly enhance the United State's access to space. After receiving his B.S. in Mechanical Engineering at the US Air Force Academy, Lt. Arritt developed components for NASA's NGST while earning his M.S. in Mechanical Engineering at the George Washington University.

Eugene Fosness is the Transition Lead for the Spacecraft Component Branch of the Space Vehicle Technologies Division at the U.S. Air Force Phillips Laboratory at Kirtland Air Force Base. Gene Fosness received a B.S. in Civil Engineering from North Dakota State University in 1981 and his M.S. 
in Civil Engineering from the University of New Mexico in 1991. He is currently employed as a Research Structural Engineer in the Space Vehicles Directorate at the U.S. Air Force Research Laboratory

Peter Wegner is a research engineer for the Air Force Research Laboratory's Space Vehicles Directorate. His research focuses on the design, fabrication, and testing of innovative structures for spacecraft and launch vehicle applications. He has a B.S. in Aerospace Engineering from the University of Arizona, a M.S. in Aerospace Engineering from Stanford University, and a Ph.D. in Mechanical Engineering from the University of Wyoming.

John Higgins is a research engineer with the Air Force Research Laboratory's Space Vehicles Directorate. His work focuses on developing next generation launch vehicle structures that improve payload accommodations and promote greater access to space. He has a B.A. in Physics and Mathematics from Vanderbilt University, an M.S. in Civil Engineering from Vanderbilt University, and a Ph.D. in Civil Engineering from Texas $A \& M$ University.

Mr. Steven Buckley is a TRW systems engineer supporting the Air Force Research Laboratory's Space Vehicles Directorate. He is involved in developing new technologies that are directly applicable to Air Force space related research and development. He is retired from the U.S. Air Force with 25 years of military service. While on active duty he was responsible for providing space lift for the launch of $16 R \& D$ satellites using three different launch vehicles. He received his B.S. in Aerospace Engineering from the University of Florida in 1983. He received an M.S. in Aerospace Engineering from the University of Dayton in 1992 